\title{
Harmony, the Union of Music and Art
}

\author{
Sama Mara \\ Musical Forms \\ UK \\ www.samamara.com \\ sama@musicalforms.com
}

\begin{abstract}
This paper discusses the creative process explored in the creation of the project $A$ Hidden Order by artist Sama Mara and composer Lee Westwood. A Hidden Order is an audio-visual project merging the worlds of Traditional Islamic Art \& Western Contemporary Composition through the universal language of mathematics. Comprised of an album, collection of artworks, a film suite interactive platform and live audio-visual performance. The project is based on a method discovered by the author that translates music into geometric artworks and vice versa, where pattern represents rhythms and colours relate to pitch. The use of this method enables unique approaches to the creation of music and art where one may create a geometric artwork entirely through the use of musical composition, or inversely create a musical piece through the visual design process or any combination of these two approaches together. This property allows for the practices, techniques and creative choices of each discipline to inspire, inform and shape the other.
\end{abstract}

Geometry. Music. Visualisation. Sonification. Space filling curves. Fractals. Music theory

\section{INTRODUCTION}

Is there an intrinsic and authentic interrelationship between music and art?

This question inspired many thinkers, artists, musicians and scientists throughout history and is the question at the root of the work presented in this paper. The perceptual and intuitive relationship seems to be shared by people in general, this is exemplified by the artist Wassily Kandinsky when he wrote "The sound of colours is so definite that it would be hard to find anyone who would express bright yellow with bass notes, or dark lake with the treble,"(Kandinsky 1914). Even in our everyday language we talk about bright notes in music, and loud colours in art, and rhythm \& pattern are almost interchangeable in both the language of music and art.

It is through the universal language of mathematics that the research presented in this paper seeks to reveal this relationship.

\subsection{Harmony}

It is understood that simple whole number ratios are the foundation of musical harmony, both in terms of rhythm and pitch. In rhythm, this can be seen in the division of a bar, for example into four beats, each of which is further subdivided perhaps into triplets. We may have other time signatures, and some may be relatively complex compared to the example given, but this basic division of time forms the foundation of rhythm. In the higher frequencies of audible pitch $20 \mathrm{hz}-20,000 \mathrm{hz}$ we find the same principle holds, that is that simple whole number ratios create harmonious sounds. Although this is by no means the end of the story as regard to harmony of sound it is a corner stone which has proved very fruitful for millennia.

Is there an equivalent visual harmony, or harmony of space? Knowledge of the golden ratio exists in contemporary culture and its harmonious qualities are exemplified in classic artworks and architecture. It is indeed a fascinating ratio numerically and geometrically, although this is only one part of a much vaster study of visual harmony. In the practice of traditional Islamic geometric art, the foundation of harmony is the circle, equally divided into the regular polygons for example triangle, square, pentagon, etc., and the natural subdivisions thereof, it is this principle that governs the method presented in here.

The golden ratio occurs naturally from the pentagon and decagon, along with a whole family of related ratios, the square and octagon reveal the silver ratio $(\sqrt{ } 2+1)$ and the triangle and hexagon are based on the $\sqrt{ } 3$ relationships. The numbers which are revealed through the subdivisions of these polygons are more complicated than their 1dimensional musical counterparts so at first it seems a difficult proposition to marry these two systems of harmony through mathematics. However, in the visual realm of geometry and by implementing modern concepts in fractal geometry we can bridge this dimensional gap between music and art. 
Once a translation is established between the creative practices of musical composition and geometric design, it allows for a cross-fertilisation of their respective qualities, creative processes and creative choices to directly inform each other, resulting in sometimes surprising results, and enabling the developments of one field to be implemented into the other. For example, the standard musical practices of layering motifs, and the structuring of a composition including contrasting sections and development of themes may be directly expressed in geometric visual form. Inversely the creative decisions in visual arts and developments in geometry such as the recent work in aperiodic tilings may inform and structure musical composition.

\section{THE METHOD}

The basic principle of the method relates the musical notes to colours, rhythm to pattern and the time signature to the symmetry of a grid.

\subsection{Pitch and Colour}

The relationship between the musical note and colour is based on the understanding of the wave nature of both sound and light. The amplitude of the wave is expressed as loudness in sound and brightness in colour, whereas frequency is related to the pitch of the sound and the hue of the colour. Within one musical octave we cover the visible spectrum plus magenta.

\subsection{Rhythm and Pattern}

The most significant development in this method is the creation of a one to one mapping between rhythm and pattern, whereby every musical rhythm has a unique visual counterpart in the form of a pattern within a given grid.

The details of the method are explained in depth in the authors publication in Bridges 2017 (Mara 2016). Here shall be illustrated only a brief overview of this method.

The origin of the method asked, if one square was to represent one unit of time (for example one beat), what would two units look like (see Figure 1). This doubling of area can be extended indefinitely.

The nested squares naturally subdivide to form a grid of equal sized cells. These cells are indexed and each cell relates to a specific period of time in music, for example the first beat is at the centre, the second beat neighbours this and so on (see Figure 2).
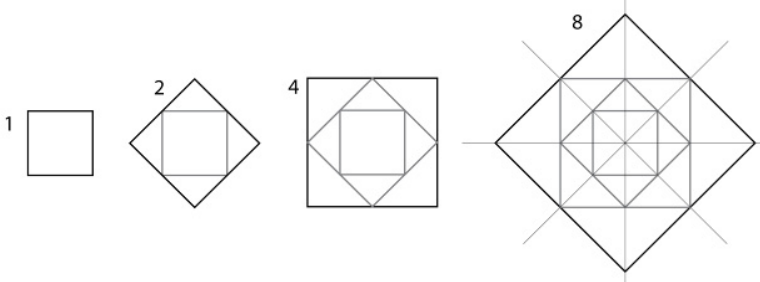

Figure 1: Progression from one square representing one beat, to double the area representing double the length of time, and the process repeated two more times to reach eight times the original area.

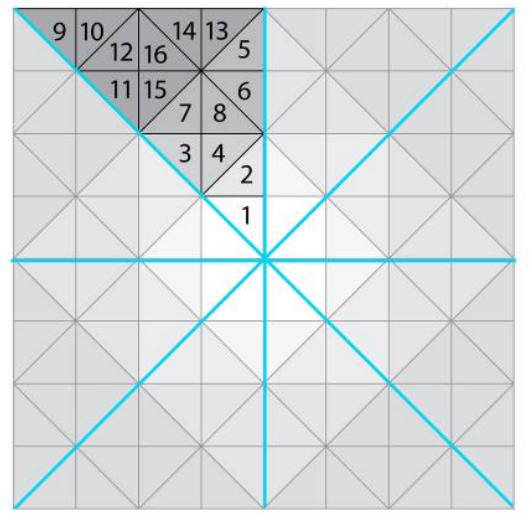

Figure 2: The square grid with indexed cells, each cell represents a beat in music. The section of the grid that is numbered is reflected and rotated to be repeated in the other seven sections around the origin.

This same approach can be applied to other grid symmetries (see Figure 3). We find that each symmetry relates to particular time-signatures (Mara 2016).

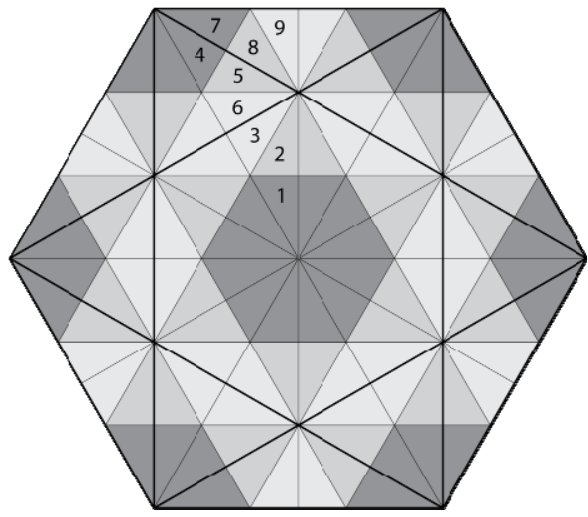

Figure 3: The hexagon with subdividing grid relating to a time signature of 9 beats per bar.

\subsubsection{Octagons and Pentagons}

Significant work has been done on tilings involving fivefold and eightfold symmetry in recent decades, with the famous Penrose-tiling and the AmmannBeenker tiling amongst others.

The resulting time signatures relating to these grids are comprised of two bar lengths (related to the two 
cell sizes in these grids) and arranged in an aperiodic sequence. In the case of the Pentagonal tiling the bar lengths are two neighbouring Fibonacci numbers for example 5 and 8 . See Mara (2016) for an example of the derivation process of sequences from these tilings. These sequences although entirely logical, geometrically beautiful and mathematically fascinating, are not at all standard in a musical context, this represents a problem in using them creatively and an opportunity to discover if these sequences have a role to play in music.

\subsection{Sound to visual}

Following is an example of implementing the method described to create a pattern from a traditional flamenco compás.



Figure 4: The hexagon with subdividing grid relating to a time signature of 9 beats per bar.

\subsection{Visual to sound}

From the above example one can see how we might read a rhythm from a pattern. By following the numbered cells in sequence and accenting the beats that are associated with the shaded cells in the pattern. Colours can likewise be read to add pitch to the resulting musical expression.

\section{COMPOSITIONAL PROCESSES}

The approaches explored in the creation of the pieces in A Hidden Order span a wide spectrum, from exclusively musical composition to pure artwork design and combinations of the two.

\subsection{Musical Composition}

Artworks: Hexagon I - Ensemble, Square Ensemble \& Hexagon II - Cello \& Percussion
On one end of the spectrum of the creative process is purely musical composition, the only requirement being to stay in a particular time signature and the use of a constant pulse, otherwise the composer has free reign as to what is created (Figure 5).

\subsection{Core motifs chosen for their visual properties}

Artwork: Triangle - Ensemble

A series of musical motifs were created and subsequently visualised and selected for their aesthetic qualities. The final composition was developed in musical form from this selection (Figure 6).

\subsection{Initial visual motifs}

Artwork: Octagon Square - Marimba

As an inversion of the previous approach, a series of visual motifs were translated into a selection of rhythms and developed into the final composition (Figure 7).

\subsection{A visual foundation}

Artworks: Octagon II - Ensemble, Octagon I - Flute \& Marimba

These pieces began as visual designs that governed the structure of the piece as well as particular motifs. This was translated into a rhythmical template and developed into the final piece. There was some going back and forth between artwork and music to arrive at a final composition. This approach represents the most balanced between its visual and aural considerations (Figure 8).

\subsection{A geometric game}

Artwork: Pentagon III - 'Roundels' Ensemble The approach towards this composition was purely geometric, exploring the properties of the tiling itself. Each radially symmetric motif within an area of the Penrose tiling was identified. These motifs appear at different scales (Figure 9b). Each instrument in the ensemble was then assigned a different size motif. Westwood was then set the task of composing motifs for each instrument whose interaction was entirely governed by the geometrical structure within this tiling (Figure 9).

\subsection{Purely Visual Design}

Artworks: Octagon III - Solo Conga - Study The only consideration in the development of this piece was the visual design, which was inspired by the muqarnas of Islamic architecture. The duo-tone nature of the design lent itself to a percussive musical rendition, finally performed on the conga (Figure 10). 



Figure 5: Hexagon II - Cello \& Percussion
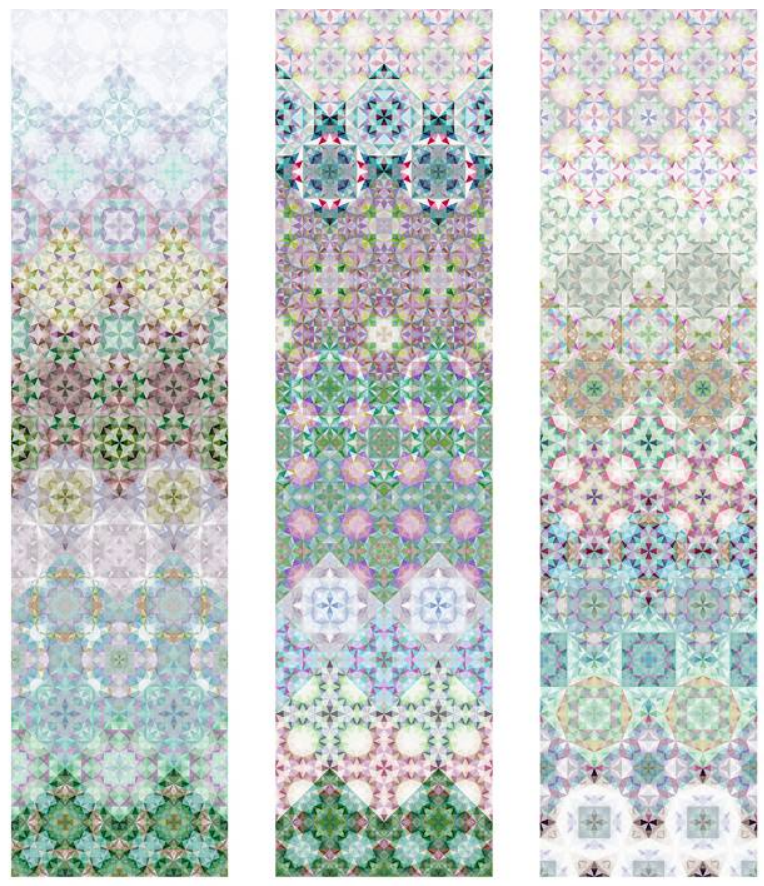

Figure 7: Octagon Square - Marimba

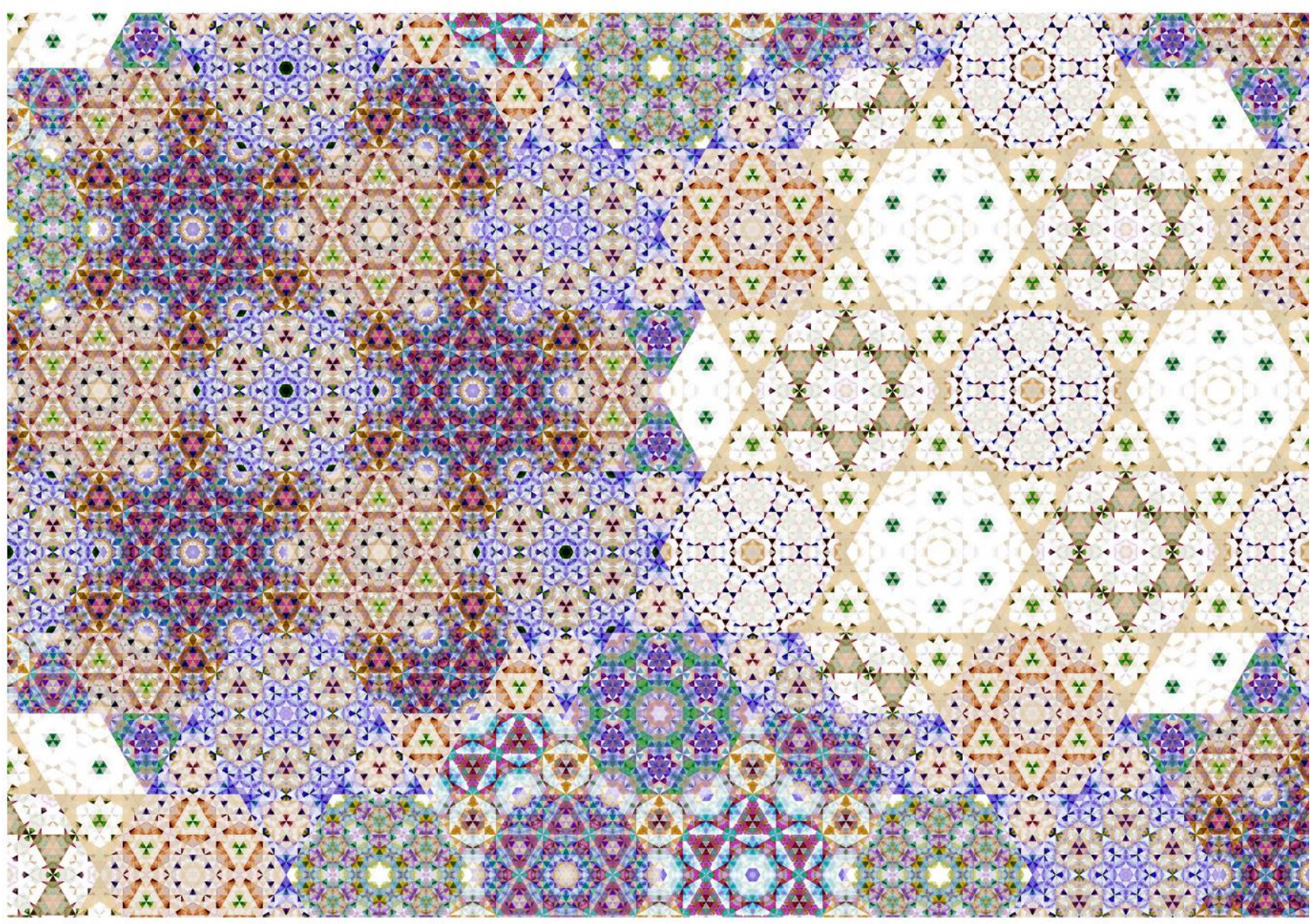

Figure 6: Detail of Triangle - Ensemble - Variation 1. The upper left area of this image displays a part of the piece where the full ensemble is performing together, creating a dense and rich design. This is in contrast to the area on the left which is very sparse, hence the large areas of white. 


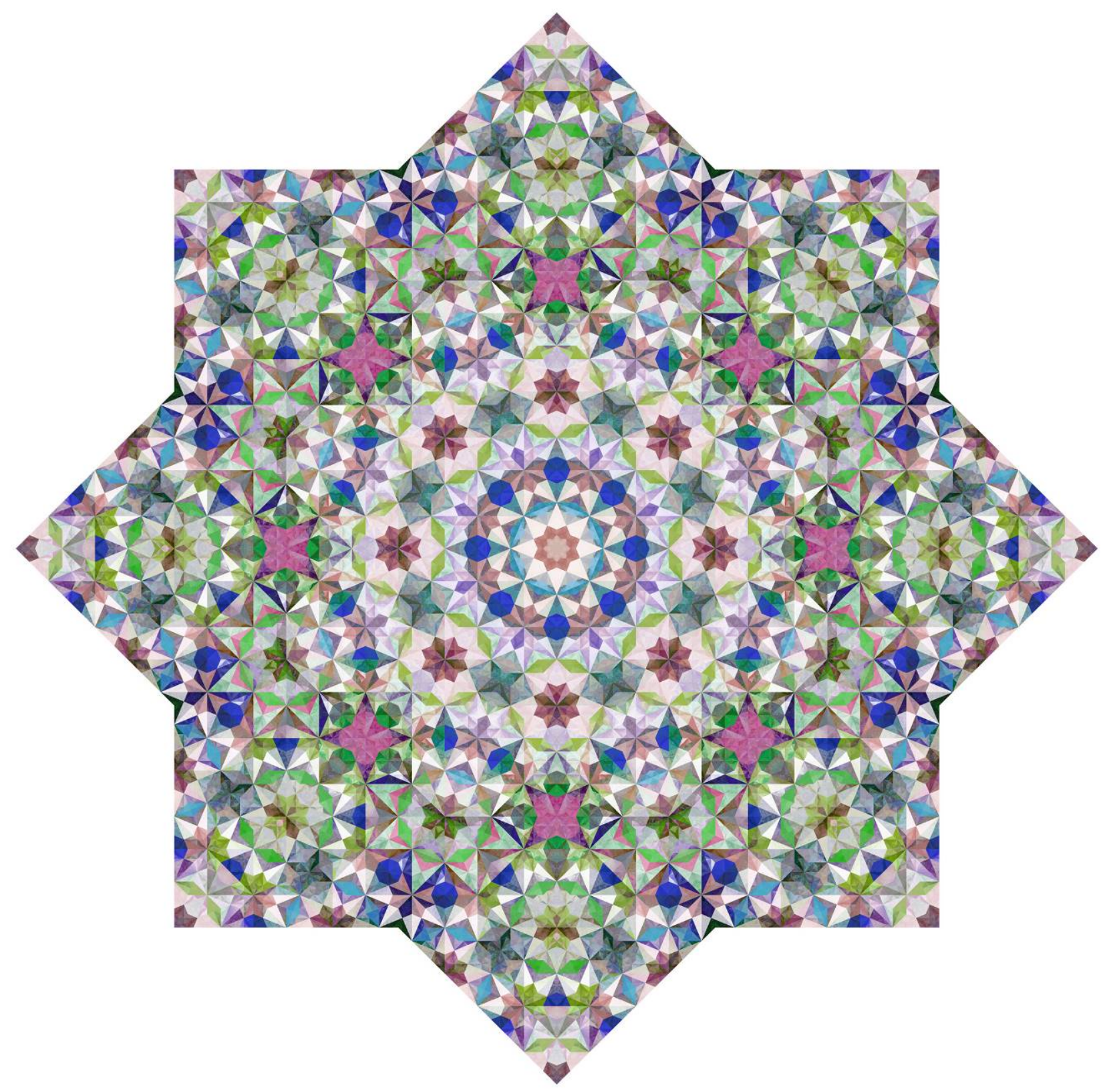

Figure 8: Octagon I - Flute \& Marimba 


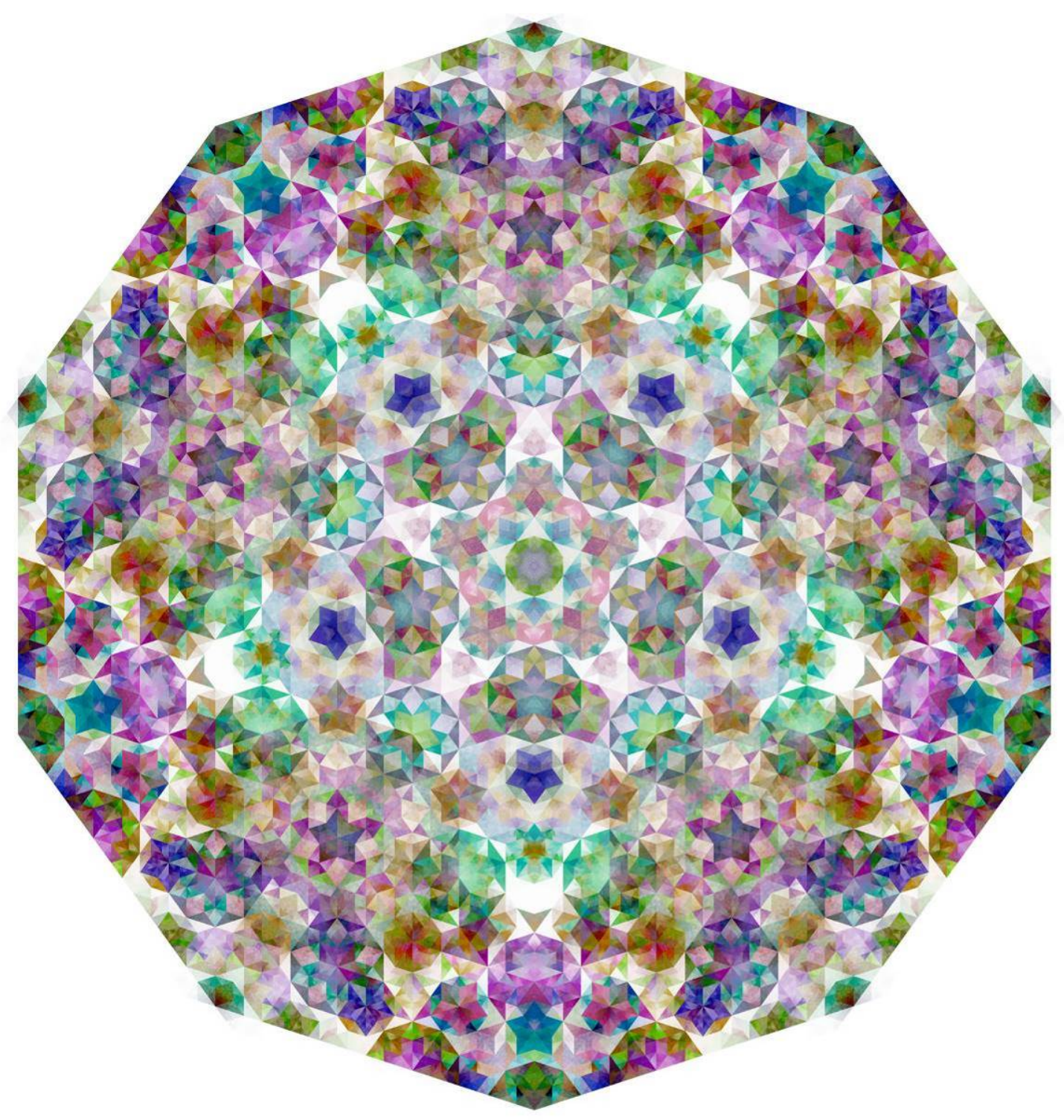

Figure 9: Pentagon III - 'Roundels' Ensemble.


Figure 9b: Penrose tiling with two scales of radially symmetric motifs identified. 


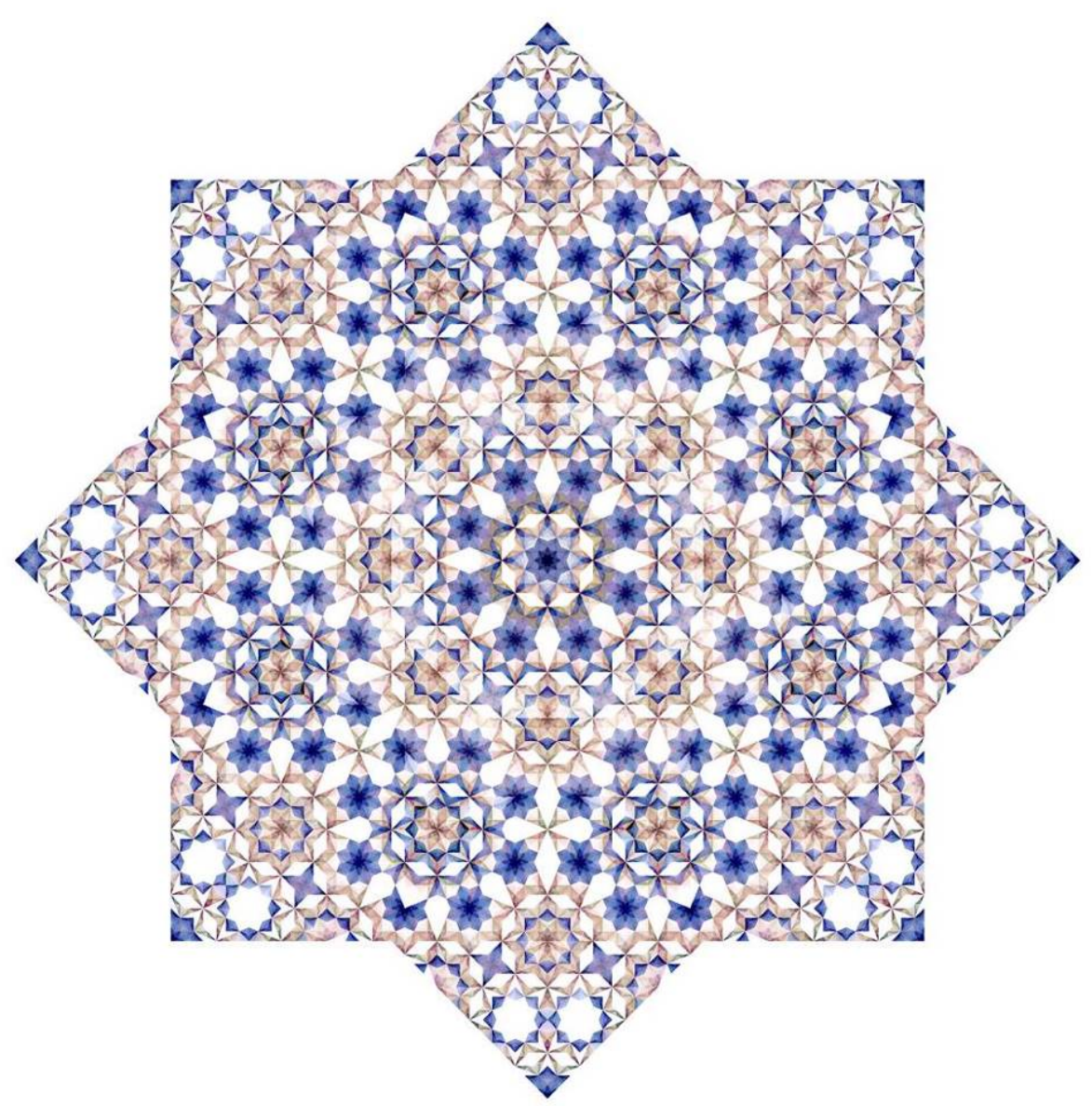

Figure 10: Octagon III - Solo Conga - Study

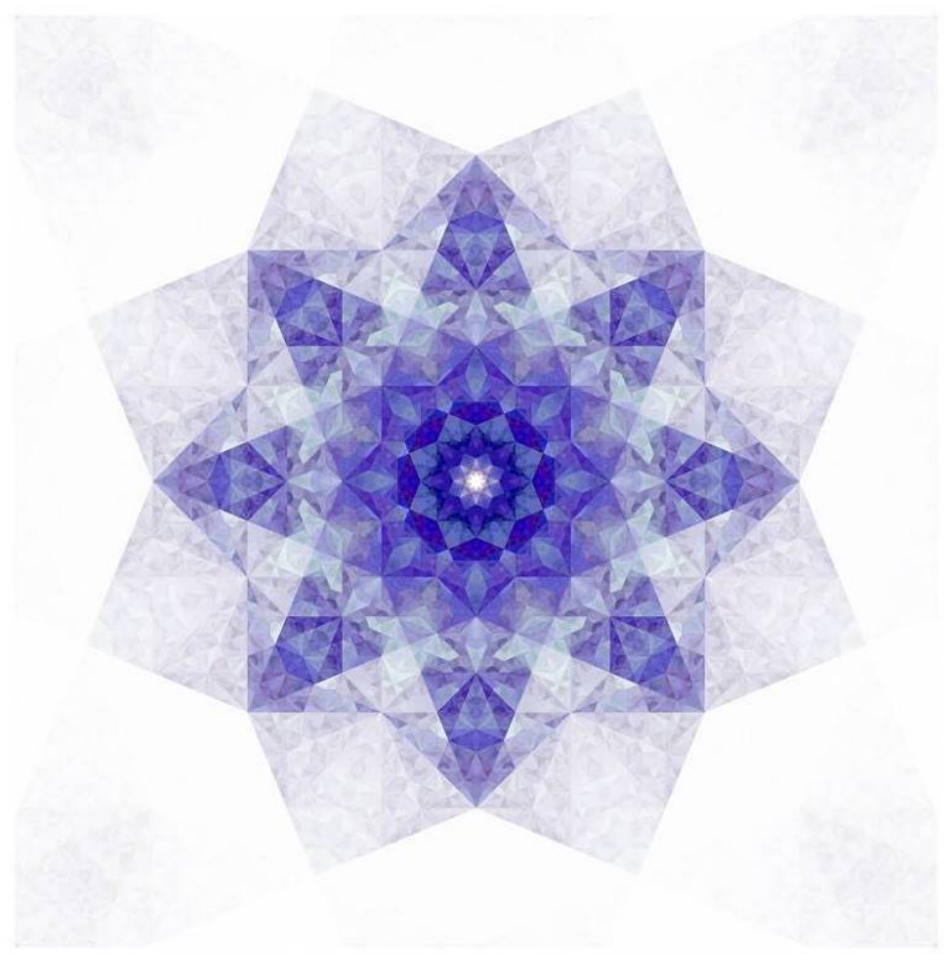

Figure 11: $D$ - Marimba. This artwork is the visualisation of single note of $D$ played on the Marimba. The white centre shows the slight delay in the performance, followed by the attack creating the rich colurs around the centre, this gradually fades out as the sound tends towards silence. The texture within the form is particular to the timbre of the sound itself. 


\section{CREATING THE FINAL WORKS}

On completion of the composition / design stage the resultant pieces were rehearsed and professionally recorded by our quartet of musicians including cor anglais, flute, marimba \& percussion and cello. Using a bespoke computer programme, built around the method described, these recordings were translated into their geometric counterparts in the form of a series of digital prints and short films.

\section{CONCLUSION}

The principles of harmony implemented are the same as those at the foundation of Islamic geometric art, thus the aesthetic of the final pieces carries a strong resonance with this tradition. There is also kaleidoscopic feel to the works as the grids themselves are constructed through a series of reflections (Mara 2016), whilst there is also a hint of fractal geometry. The final result spans different traditions and visual styles but is not limited to any one in particular.

\subsection{Aperiodic sequences}

The musical freedom allowed by the simple grids and related time signatures of the hexagon, square and triangle meant that Westwood was allowed full freedom to compose using standard musical composition approaches. The resultant artworks were to our perception instantly aesthetically pleasing and we felt no need to further develop the pieces for their visual properties.

The restrictions caused by the octagonal and pentagonal grids were a challenge to work within and the creative process felt stifled and at times frustrating. Within these aperiodic grids and time signatures the pieces that were most successful started from a more developed and specific visual template which was then translated into a rhythmical structure to be developed into the final musical piece.

The positive aspect of working with these aperiodic grids was the opportunity of creating music within unorthodox time signatures based on aperiodic sequences from modern mathematics. The music sounds at times very natural and to my ear very pleasing. The irregularity of the section lengths lends unpredictability to the music and a fluidity to the structure, however this irregularity is not random or unorganised. Some listeners have reported that they find the music challenging. It is not clear if this is due to the style of contemporary classical music in general, or the aperiodic structure of some of the pieces themselves.
A peculiar part of the creative process was that sometimes the feedback given to Westwood after developing the piece from the visual aspect would be very specific. Composers are used to general feedback pertaining to mood, dynamics, and lengths of sections, whereas the aim of my feedback might be to reveal a certain visual motif so the request could be to alter the pitch of a certain beat in a particular set of bars. Mostly we could find solutions to create satisfying results.

What was most satisfying was the ability to bring approaches from musical composition directly into the visual realm. Particularly those aspects of layering of different motifs and creating a complex final texture Figure 6 . The results here although entirely under the control of the musical input would be very hard to design from a visual approach alone, as there are four instruments performing as an ensemble including the polyphonic Marimba.

Also, a surprise is how at times neighbouring sections would interact with each other as the patterns and forms stretch across the boundaries of their individual tiles, merging seamlessly and creating a continuous rich tapestry of form and colour, where it is difficult to distinguish where one section ends and another begins (see Figure 6).

Due to the detailed analyses enabled using the computer, the final artworks display the dynamics of the original performance with the variations in accents, the attack and decay of the sounds and the timbre of the instruments themselves is visualised as the texture within the patterns (see Figure 11).

The works created in A Hidden Order are very much an exploration and were being developed even as the method itself was still being honed, as such the full potential possible has hardly been explored. We are looking forward to future projects which are more directed, where we are not so much exploring the new concepts and approaches but using the more successful approaches we have discovered to craft more refined and beautiful pieces.

\section{REFERENCES}

Kandinsky, W. (1914) Concerning the Spiritual in Art. Dover Publications Inc. (1977), p. 25.

Mara, S. (2016) The Rhythm of a Pattern. Bridges Finland 2016, The University of Jyväskylä, 9-13 August, pp. 309-316. Tessellations Publishing, Phoenix, Arizona, USA. 